\title{
Aryl Chalcones as Efficient Precursors for Deriving Oxazine: Solvent-free Synthesis and Antimicrobial Activities of some Oxazine-2-amines
}

\author{
G. Thirunarayanan ${ }^{1 *}$, R. Sundararajan ${ }^{2}$, R. Arulkumaran ${ }^{2}$ \\ ${ }^{1}$ Department of Chemistry, Annamalai University, Annamalainagar - 608002, India \\ ${ }^{2} \mathrm{PG}$ and Research Department of Chemistry, Government Arts College, C-Mutlur, \\ Chidambaram - 608102, India \\ *E-mail address: drgtnarayanan@gmail.com
}

\begin{abstract}
A series of some oxazine derivatives has been synthesised by fly-ash: $\mathrm{H}_{2} \mathrm{SO}_{4}$ catalyzed solventfree cyclization of aryl chalcones and urea under microwave irradiation. The yields of the oxazines were more than $85 \%$. The synthesised oxazines were characterized by their physical constants, analytical and spectroscopic data. The antimicrobial activities of these oxazines have been studied using Bauer-Kirby method.
\end{abstract}

Keywords: Oxazine amines; Chalcones; fly-ash: $\mathrm{H}_{2} \mathrm{SO}_{4}$; Environmentally benign reaction; antimicrobial activities

\section{INTRODUCTION}

Oxazines are a six membered heterocyclic compounds possess one oxygen and one nitrogen atom. Depend upon the relative position of these tow atoms and the double bond, these molecule exists many isomeric structures such as 1,2 or 1,3 or 1,4 oxazines [1].

These oxazines were medicinally important due to the presence of oxygen, nitrogen heteroatoms along with a double bonds in their structural moieties [2]. The important medicinal activities of these oxazines are anti-bacterial [2-4], anti-fungal [2-4], antiplasmodial [5], anti-cancer [6], anti-depressants [7], cytotoxicity [8], anti-osteoplastic [9], anti-tumour [10], anti-oxidant [11], anti-tuberculosis [12], anti-neoplastic [13], antagonists [14], anti-inflammatory [15], anti-infectants [16], IKB kinase beta [17] and PTP-1B inhibition [18].

These oxazine derivatives were applied for improving the super resolution microscope [19], synthesis of eosinophils [20] identification and separation of neutrophils [21]. Many oxazine derivatives were used as a dyes [22]. Numerous solvent assisted and solvent-free synthetic methods were available for synthesis of oxazine derivatives [23]. Now-a-days scientists, organic chemists are interested for solvent-free synthesis [3,24-32]. Hetero Diels- 
alder reaction [2], ring closure [33], Betti base induced condensation [24-32], Mannich type condensation-cyclization [3] and cyclization of chalcones [4] were used for synthesis of oxazine derivatives. Verma et. al., [24-32] have synthesised some benzoxazine/oxazine fused isoquinolines and naphthyridines by solvent-free method. Elarfi and Al-difar [4] have synthesised some 1,3-oxazine derivatives by solvent-assisted method from chalacones and urea. More than $75 \%$ yield of dihydro- ${ }^{2} \mathrm{H}$-benzo- and naphtho-1,3-oxazine derivatives were prepared by Mathew et al. [3] using eco-friendly method.

Efficient synthesis of some 1,3-oxazine-4-thiones were synthesised by $\mathrm{N}$ methylimidazole promoted solvent-free conditions. Sapkal et al., have studied the role of ammonium acetate for solvent-free synthesis of 1,3-disubstituted-2,3-dihydro- ${ }^{1} \mathrm{H}$-naphyl oxazines [24-32] Within the above view, there is no information available in the literature for the solvent-free synthesis and the study of antimicrobial activities of $9 H$-fluorene-2-yl based oxazine 2-amine derivatives.

Therefore the authors have taken effort to synthesize some $9 H$-fluorene-2-yl based oxazine 2-amine derivatives and study the antimicrobial activities using Bauer-Kirby method.

\section{EXPERIMENTAL}

\section{1. General}

All chemicals were used in this study were purchased from Sigma-Aldrich and Merck Chemical companies. Mettler FP51 melting point apparatus was used for determining the melting point of all synthesized oxazines in open glass capillaries and are uncorrected. The AVATAR-300 Fourier transform spectrophotometer was used for recording infrared spectra $\left(\mathrm{KBr}, 4000-400 \mathrm{~cm}^{-1}\right)$ of all oxazines in $\mathrm{KBr}$ disc.

The Bruker AV400 series type NMR spectrometer was utilized for recording NMR spectra of all oxazines, operating at $400 \mathrm{MHz}$ for ${ }^{1} \mathrm{H}$ and100 $\mathrm{MHz}$ for ${ }^{13} \mathrm{C}$ spectra in $\mathrm{CDCl}_{3}$ solvent using TMS as internal standard. Mass spectra of all synthesised oxazines were recorded on SHIMADZU mass spectrometer using chemical ionization technique.

\section{Preparation of fly-ash: $\mathrm{H}_{2} \mathrm{SO}_{4}$ catalyst}

The fly-ash: $\mathrm{H}_{2} \mathrm{SO}_{4}$ catalyst was prepared according to literature procedure [34].

\section{Synthesis of 4-(aryl)-5,6-dihydro-6-(substituted phenyl)-4H-1,3-oxazine-2-amines}

An appropriate equi-molar quantities of chalcones $(2 \mathrm{mmol})$, urea $(2 \mathrm{mmol})$ and $0.2 \mathrm{~g}$ of fly-ash: $\mathrm{H}_{2} \mathrm{SO}_{4}$ were taken in a $50 \mathrm{~mL}$ beaker, closed with the lid.

This mixture was subjected to microwave irradiation for 2-4 minutes at $650 \mathrm{~W}$ (Scheme 1) (Samsung, Microwave Oven, 100-700 W). After completion of the reaction, dichloromethane $(20 \mathrm{~mL})$ was added, followed by simple filtration.

The solution was concentrated and purified by re-crystallization. The synthesized oxazines were characterized by their physical constants, IR, ${ }^{1} \mathrm{H}$ and ${ }^{13} \mathrm{C}$ NMR and Mass spectral data. Analytical and Mass spectral data are presented in Table 1. 
<smiles>[R]C(=O)/C=C(\[R])[FH+]</smiles>

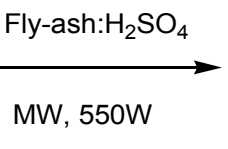<smiles>[R]C1CC([R])OC(N)=N1</smiles><smiles>[R]=CCCCCCCCC</smiles>

Scheme 1. Synthesis of 4-aryl-5,6-dihydro-6-(substituted phenyl)-4H-1,3-oxazine-2-amines by fly-ash: $\mathrm{H}_{2} \mathrm{SO}_{4}$ catalyzed cyclization of aryl chalcones and urea under microwave irradiation.

Table 1. Analytical, physical constants, yield and mass fragment of 4-aryl-5,6-dihydro-6(substituted phenyl)-4H-1,3-oxazine-2-amines.

\begin{tabular}{|c|c|c|c|c|c|c|}
\hline Entry & $\mathbf{R}$ & $\mathbf{R}^{\prime}$ & M. W. & $\begin{array}{c}\text { Yield } \\
(\%)\end{array}$ & $\begin{array}{l}\text { m.p. } \\
\left({ }^{\circ} \mathbf{C}\right)\end{array}$ & Mass $(\mathbf{m} / \mathbf{z})$ \\
\hline 1 & & & 252 & 88 & $\begin{array}{l}0 \\
\frac{1}{1} \\
\frac{1}{2} \\
\end{array}$ & $\begin{array}{l}252 \mathrm{M}^{+}, 236 \\
175,160,84 \\
77,43,42,16\end{array}$ \\
\hline 2 & & & 268 & 85 & 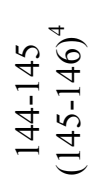 & $\begin{array}{c}268 \mathrm{M}^{+}, 252, \\
251,236,175, \\
160,99,93,84, \\
77,43,42,16\end{array}$ \\
\hline 3 & & & 295 & 87 & 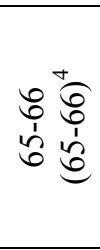 & $\begin{array}{c}295 \mathrm{M}^{+}, 280, \\
265,279,251, \\
236,175,160, \\
118,84,77,44, \\
43,42,30,16, \\
15\end{array}$ \\
\hline 4 & & & 282 & 89 & $\begin{array}{l}\text { İ } \\
\text { తి }\end{array}$ & $\begin{array}{c}282 \mathrm{M}^{+}, 266, \\
251,236,205, \\
190,175,160, \\
107,91,84,77, \\
43,42,31,16\end{array}$ \\
\hline
\end{tabular}




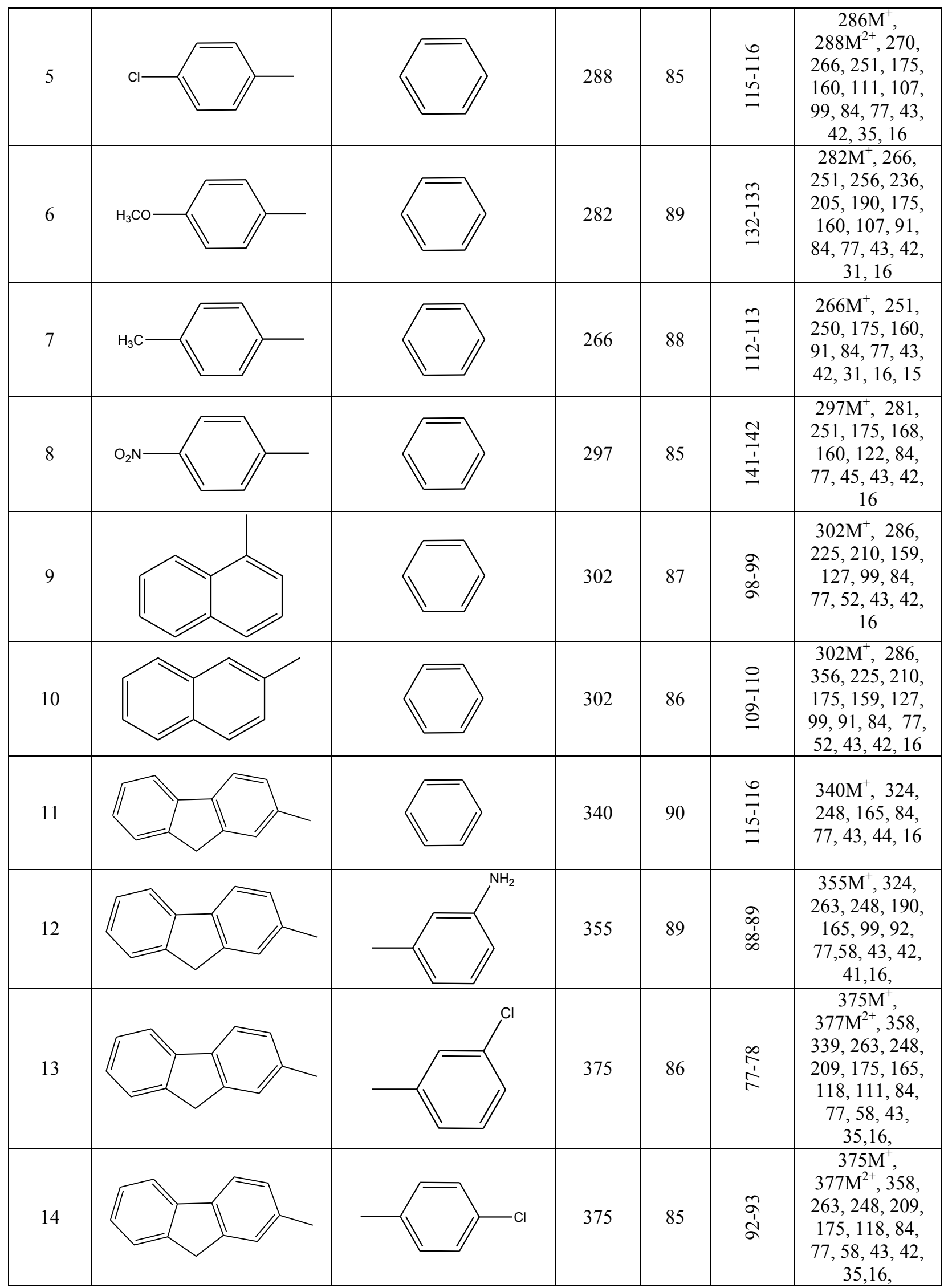




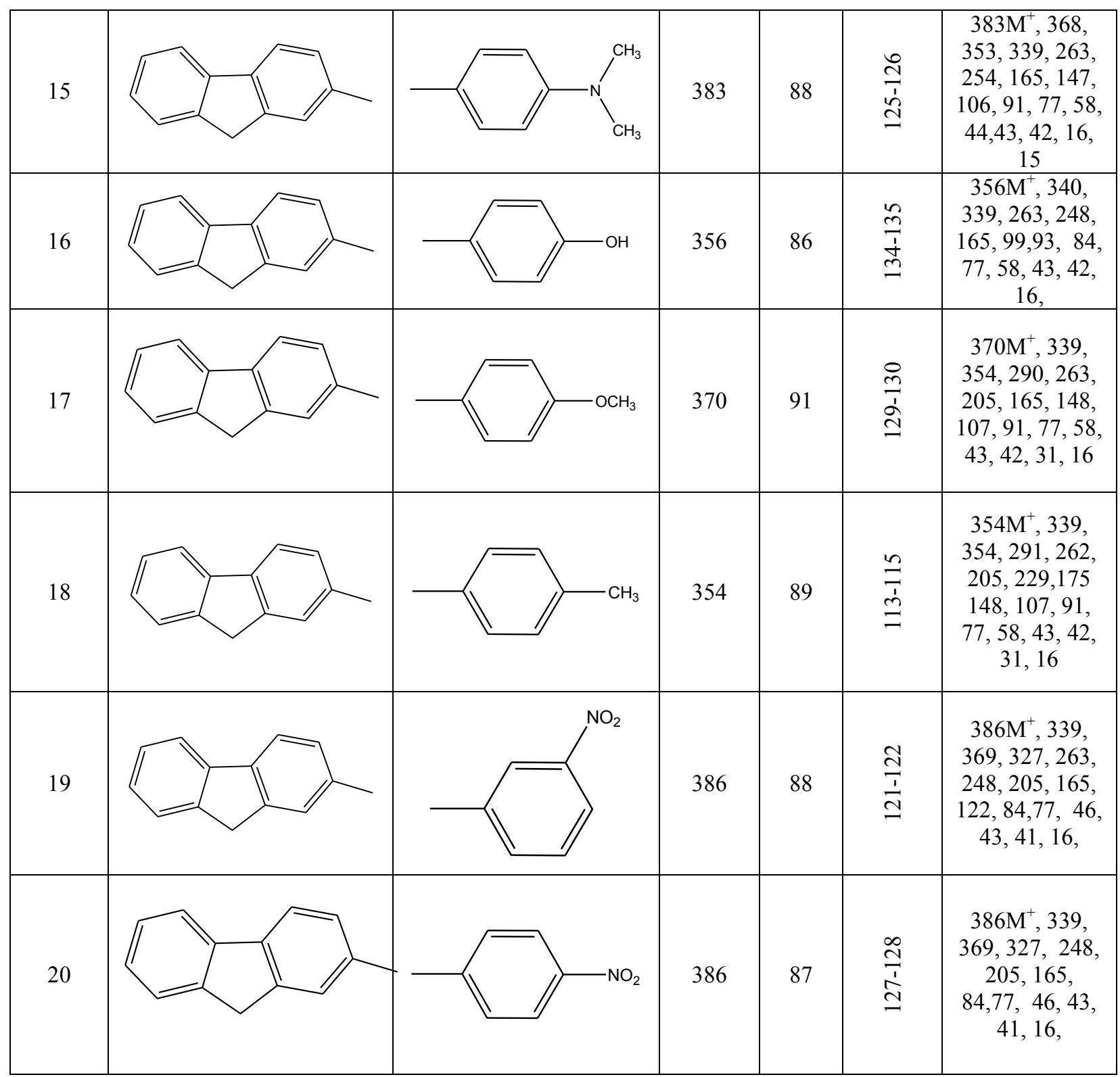

\section{RESULTS AND DISCUSSION}

In our organic chemistry research laboratory, the author attempt to synthesize oxazine derivatives by cyclization of chalcones possess electron with-drawing as well as electron donating group as substituents, urea and in the presence of acidic catalyst fly-ash: $\mathrm{H}_{2} \mathrm{SO}_{4}$ using microwave irradiation. Hence the authors have synthesized some substituted 1,3oxazine derivatives by the cyclization of 2 mmole of chalcone, 2 mmole of urea under microwave irradiation with $0.4 \mathrm{~g}$ of fly-ash: $\mathrm{H}_{2} \mathrm{SO}_{4}$ catalyst at $550 \mathrm{~W}$ for 4-6 minutes (Samsung Grill, GW73BD Microwave oven, 230 V A/c, 50Hz, 2450 Hz, 100-750 W (IEC705), (Scheme 1). During the course of this reaction fly-ash: $\mathrm{H}_{2} \mathrm{SO}_{4}$ catalyses cyclization between chalcones and urea followed by rearrangement gave the 1,3-oxazine amines. The yield of the oxazine in this reaction are more than $80 \%$. The chalcone containing electron donating substituent $\left(\mathrm{OCH}_{3}\right)$ gave higher yields than electron-withdrawing (halogens, $\mathrm{NO}_{2}$ ) 
substituents. Further we have investigated this cyclization reaction with equimolar quantities of the styryl 9H-fluorennyl ketone (entry 10 ) and urea under the same condition as above. In this reaction the obtained yield was $90 \%$. The effect of catalyst on this reaction was studied by varying the catalyst quantity from $0.1 \mathrm{~g}$ to $1 \mathrm{~g}$. As the catalyst quantity is increased from $0.1 \mathrm{~g}$ to $1 \mathrm{~g}$, the percentage of yield of product is increased from 84 to $90 \%$. Further increase in the catalyst amount beyond $0.4 \mathrm{~g}$, there is no significant increase in the percentage of the product. The effect of catalyst loading is shown in Fig. 1. The optimum quantity of catalyst loading was found to be $0.4 \mathrm{~g}$. The reusability of this catalyst was

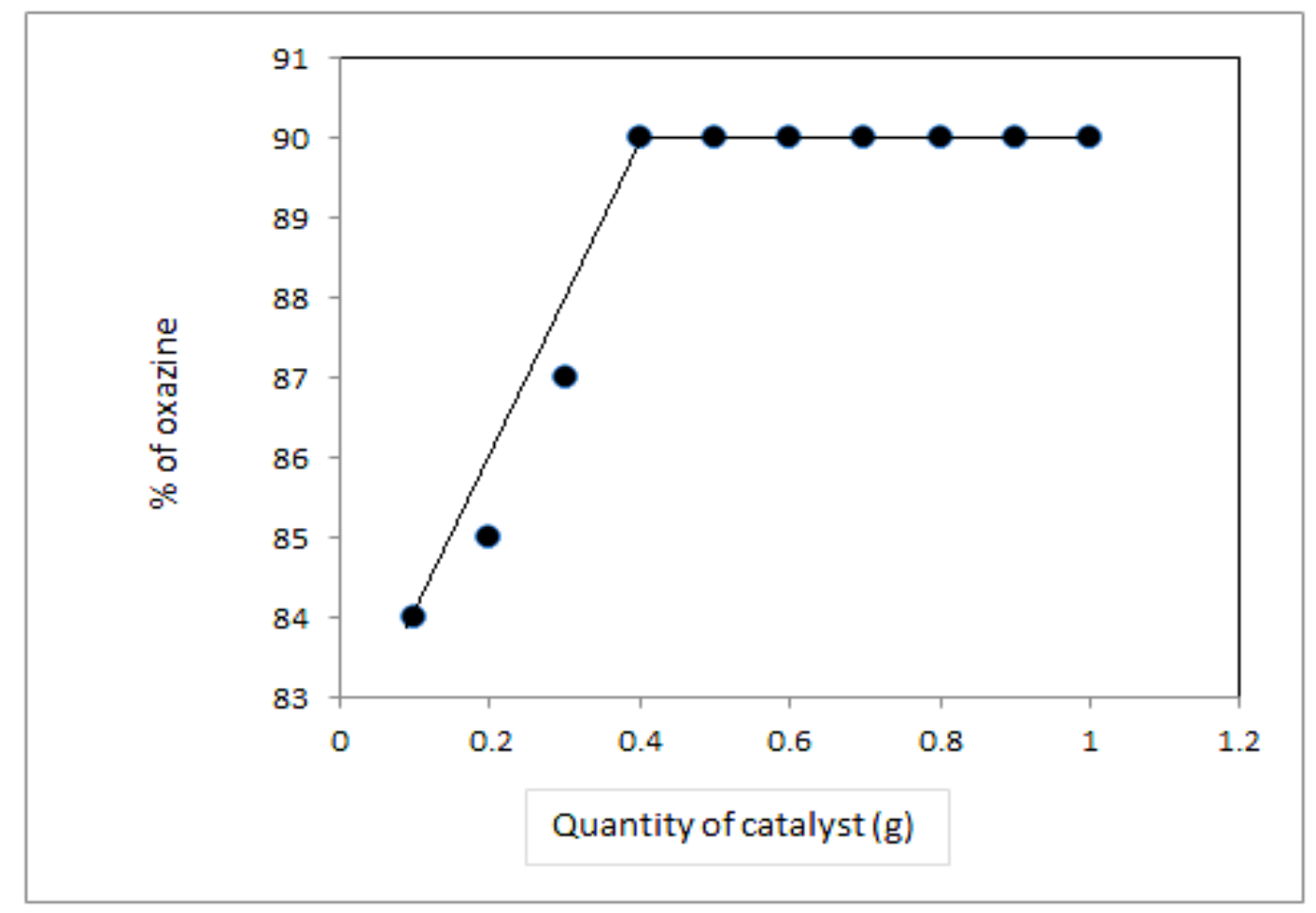

Fig. 1. Effect of catalyst loading.

The reusability of this catalyst was studied for the cyclization of styryl $9 H$-fluorenyl ketone, and urea (entry 10) is presented in Table 2. From the Table 2, first two runs gave 90 $\%$ product. The third, fourth and fifth runs of reactions gave respectively the yields $89.5 \%$, $89.5 \%$ and $93 \%$ of oxazines.

There was no appreciable loss in its effect of catalytic activity observed up to fifth run. The effect of solvents on the yield was also studied with methanol, ethanol, dichloromethane and tetrahydrofuran from each component of the catalyst (entry 10).

Table 2. Reusability of fly-ash: $\mathrm{H}_{2} \mathrm{SO}_{4}$ catalyst on cyclization cum acetylation of styryl 9H-fluorene-2yl ketone $(2 \mathrm{mmol})$ with urea $(2 \mathrm{mmol})$ under microwave irradiation (entry 11).

\begin{tabular}{|c|c|c|c|c|c|}
\hline Run & 1 & 2 & 3 & 4 & 5 \\
\hline Yield & 90 & 90 & 89.5 & 89.5 & 89 \\
\hline
\end{tabular}


Similarly the effect of microwave irradiation was studied on each component of the catalyst. The effect of solvents on the yield of oxazine derivatives was presented in Table 3 . From the table highest yield of oxazine obtained from the cyclization of chalcones and urea with the catalyst fly-ash: $\mathrm{H}_{2} \mathrm{SO}_{4}$ in microwave irradiation. The infrared and $\mathrm{nmr}$ spectroscopic data of these 1-acetyl pyrazolines are summarized in Table 4.

Table 3. The effect of solvents in conventional heating and without solvent in microwave irradiation on yield of oxazine amine (entry 11).

\begin{tabular}{|c|c|c|c|c|c|c|c|c|c|c|c|c|c|c|}
\hline \multicolumn{12}{|c|}{ Solvents } & \multirow{2}{*}{\multicolumn{3}{|c|}{$\begin{array}{l}\text { Microwave } \\
\text { irradiation }\end{array}$}} \\
\hline \multicolumn{3}{|c|}{$\mathrm{MeOH}$} & \multicolumn{3}{|c|}{$\mathrm{EtOH}$} & \multicolumn{3}{|c|}{ DCM } & \multicolumn{3}{|c|}{ THF } & & & \\
\hline FA & SA & FASA & FA & SA & FASA & FA & SA & FASA & FA & SA & FAPA & FA & SA & FASA \\
\hline 62 & 43 & 78 & 60 & 45 & 85 & 64 & 42 & 85 & 65 & 46 & 87 & 70 & 73 & 90 \\
\hline
\end{tabular}

Table 4. Infrared and NMR spectroscopic data of 4-aryl-5,6-dihydro-6(substituted phenyl)-4H-1,3oxazine-2-amines.

\begin{tabular}{|c|c|c|c|c|c|c|c|c|c|c|c|c|c|}
\hline \multirow{2}{*}{ Entry } & \multicolumn{4}{|c|}{$\operatorname{IR}\left(v, \mathrm{~cm}^{-1}\right)$} & \multicolumn{7}{|c|}{${ }^{1} \mathbf{H}(\delta, p p m)$} & \multicolumn{2}{|c|}{$\begin{array}{c}{ }^{13} \mathrm{C} \\
(\delta, \mathrm{ppm})\end{array}$} \\
\hline & 号 & $\pi^{7}$ & $\begin{array}{l}0 \\
0 \\
0 \\
\text { U }\end{array}$ & 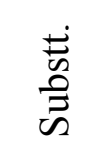 & 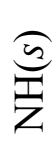 & 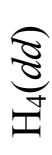 & 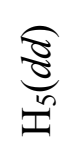 & $\underset{\substack{\mathfrak{g} \\
\text { in }}}{ }$ & 胥 & & $\begin{array}{l}\tilde{E}_{0} \\
\stackrel{0}{E} \\
\tilde{n}\end{array}$ & $\mathcal{U}^{N}$ & $U^{+}$ \\
\hline 1 & $\underset{n}{\tilde{n}}$ & $\begin{array}{l}\infty \\
\stackrel{2}{2}\end{array}$ & $\stackrel{+}{\beth}$ & 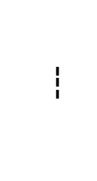 & $\stackrel{\substack{n \\
m}}{\text { m }}$ & $\begin{array}{l}\text { त̂ } \\
\text { i } \\
\text { in }\end{array}$ & 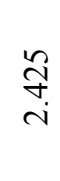 & $\underset{\sim}{\stackrel{J}{\sim}}$ & $\begin{array}{l}\hat{n} \\
\stackrel{y}{+}\end{array}$ & 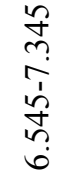 & 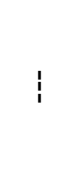 & $\begin{array}{l}m \\
\hat{n} \\
\hat{b} \\
-1\end{array}$ & $\begin{array}{l}\stackrel{0}{n} \\
i n\end{array}$ \\
\hline 2 & $\begin{array}{l}\mathbb{b} \\
\stackrel{n}{n} \\
m\end{array}$ & 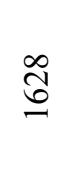 & $\stackrel{n}{\stackrel{n}{I}}$ & 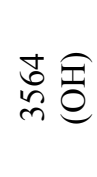 & $\stackrel{\text { ते }}{i}$ & $\begin{array}{l}\infty \\
\stackrel{n}{n} \\
i\end{array}$ & $\begin{array}{l}n \\
\stackrel{n}{0} \\
i \\
i\end{array}$ & 록 & $\stackrel{\vec{n}}{\stackrel{+}{+}}$ & 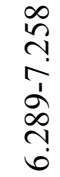 & 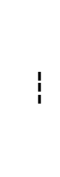 & 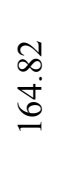 & $\frac{0}{n}$ \\
\hline
\end{tabular}




\begin{tabular}{|c|c|c|c|c|c|c|c|c|c|c|c|c|c|}
\hline 3 & $\begin{array}{l}0 \\
\text { N } \\
m\end{array}$ & $\stackrel{\nabla}{\sigma}$ & d & 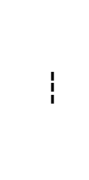 & $\underset{\sim}{\stackrel{\nabla}{v}}$ & $\stackrel{\vec{q}}{\stackrel{i}{i}}$ & $\begin{array}{l}\stackrel{\infty}{g} \\
\stackrel{\sim}{i} \\
i\end{array}$ & ì & $\stackrel{\vec{n}}{\stackrel{r}{*}}$ & $\begin{array}{l}\infty \\
\hat{n} \\
\hat{n} \\
\infty \\
\infty \\
n \\
0\end{array}$ & 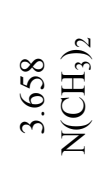 & \begin{tabular}{l}
$\tilde{n}$ \\
\multirow{\sigma}{\sigma}{}
\end{tabular} & $\begin{array}{l}\text { ते } \\
\text { in }\end{array}$ \\
\hline 4 & $\frac{\nabla}{n}$ & $\stackrel{0}{\sigma}$ & 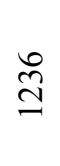 & 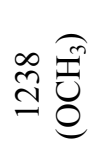 & $\begin{array}{l}\overrightarrow{0} \\
\stackrel{n}{i}\end{array}$ & $\underset{i}{\stackrel{i}{J}}$ & $\stackrel{\text { I }}{\stackrel{n}{n}}$ & ત્ત & $\begin{array}{l}\widetilde{\sigma} \\
\sigma \\
+\end{array}$ & $\begin{array}{l}\hat{\infty} \\
o \\
\hat{1} \\
\hat{1} \\
\end{array}$ & 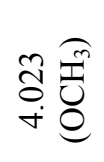 & 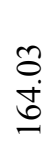 & $\begin{array}{l}\stackrel{\infty}{\sim} \\
i \\
i\end{array}$ \\
\hline 5 & $\begin{array}{l}0 \\
\tilde{n} \\
n \\
n\end{array}$ & हे & $\begin{array}{l}n \\
\stackrel{2}{]}\end{array}$ & 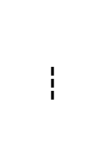 & $\stackrel{m}{\stackrel{n}{i}}$ & $\frac{\infty}{a}$ & in & $\stackrel{M}{\vec{i}}$ & $\underset{+}{\stackrel{J}{r}}$ & $\frac{\overrightarrow{\grave{N}}}{\stackrel{+}{ \pm}}$ & 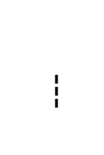 & $\begin{array}{l}= \\
+ \\
\mathbb{J}\end{array}$ & $\begin{array}{l}\text { oे } \\
\text { in }\end{array}$ \\
\hline 6 & $\begin{array}{l}\tilde{n} \\
\tilde{n} \\
m\end{array}$ & $\overline{\widetilde{V}}$ & $\stackrel{\infty}{\beth}$ & 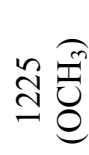 & $\underset{\hat{~}}{\stackrel{N}{~}}$ & 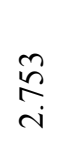 & ते & $\stackrel{\vec{N}}{i}$ & $\underset{\sim}{\tilde{\sigma}}$ & $\begin{array}{l}\tilde{n} \\
\stackrel{n}{?} \\
\frac{1}{\infty}\end{array}$ & $\begin{array}{l}\hat{\alpha} \\
\hat{\sigma}] \\
\text { ஸे }\end{array}$ & 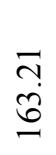 & $\stackrel{\overbrace{}}{\stackrel{i}{n}}$ \\
\hline 7 & $\begin{array}{l}0 \\
n \\
n \\
n\end{array}$ & $\tilde{\hat{n}}$ & $\underset{\Xi}{\stackrel{\Xi}{\Xi}}$ & $\mid$ & $\stackrel{\hat{a}}{i}$ & $\begin{array}{l}\hat{\delta} \\
\infty \\
i\end{array}$ & $\stackrel{\stackrel{n}{\sim}}{\underset{n}{n}}$ & $\underset{i}{\stackrel{N}{I}}$ & $\begin{array}{l}\hat{\sigma} \\
\dot{+} \\
+\end{array}$ & $\begin{array}{l}\frac{n}{n} \\
\stackrel{n}{a} \\
\frac{1}{a}\end{array}$ & 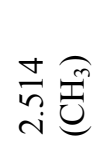 & $\begin{array}{l}\stackrel{+}{*} \\
\dot{J}\end{array}$ & $\begin{array}{l} \pm \\
\infty \\
i \\
n\end{array}$ \\
\hline 8 & $\begin{array}{l}\infty \\
\tilde{n} \\
n\end{array}$ & $\underset{\Xi}{\mathbb{J}}$ & $\begin{array}{l}n \\
\text { d్ } \\
\text { s. }\end{array}$ & 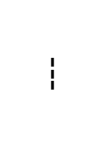 & $\frac{\vec{n}}{\vec{n}}$ & $\begin{array}{l}\hat{a} \\
\infty \\
i\end{array}$ & $\begin{array}{l}\stackrel{0}{\vartheta} \\
\text { i } \\
i\end{array}$ & $\underset{\widetilde{N}}{\tilde{i}}$ & $\begin{array}{l}\stackrel{尺}{+} \\
\dot{+}\end{array}$ & $\begin{array}{l}n \\
0 \\
\infty \\
0 \\
\\
\end{array}$ & $\mid$ & \begin{tabular}{l} 
त̂ \\
\multirow{n}{0}{}
\end{tabular} & $\stackrel{\infty}{\stackrel{\infty}{\sim}}$ \\
\hline 9 & $\begin{array}{c}\tilde{n} \\
\tilde{n}\end{array}$ & $\begin{array}{l}\stackrel{D}{2} \\
\stackrel{2}{n}\end{array}$ & $\stackrel{\Xi}{\beth}$ & 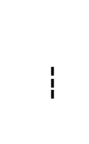 & 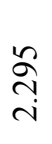 & 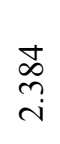 & 空 & 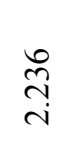 & 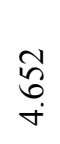 & 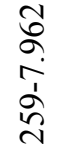 & 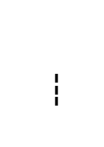 & $\begin{array}{l}\text { बे } \\
\text { +े }\end{array}$ & $\stackrel{0}{n}$ \\
\hline 10 & స్ & $\stackrel{\infty}{\stackrel{\circ}{n}}$ & $\stackrel{n}{\beth}$ & $\mid$ & ते & $\begin{array}{l}\overline{0} \\
\text { i্} \\
i\end{array}$ & $\overrightarrow{\widetilde{N}}$ & 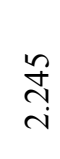 & $\begin{array}{l}\tilde{\sim} \\
\stackrel{+}{+}\end{array}$ & $\begin{array}{l}\tilde{n} \\
\infty \\
\hat{1} \\
\hat{n} \\
\text { ñ }\end{array}$ & $\mid$ & $\begin{array}{l}0 \\
0 \\
0 \\
0\end{array}$ & $\begin{array}{l}\overrightarrow{0} \\
\text { in }\end{array}$ \\
\hline 11 & 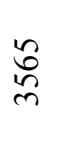 & $\frac{m}{6}$ & 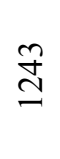 & $\mid$ & $\frac{n}{i}$ & $\begin{array}{l}\hat{\sigma} \\
\text { i } \\
\text { in }\end{array}$ & $\stackrel{\sqrt[n]{n}}{i}$ & 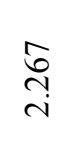 & $\stackrel{\grave{\hat{े}}}{+}$ & $\begin{array}{l}\hat{\sigma} \\
\dot{0} \\
\dot{1} \\
\dot{v} \\
\dot{v}\end{array}$ & 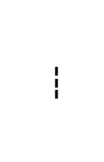 & 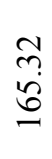 & $\begin{array}{l}\text { ते } \\
\text { ñ }\end{array}$ \\
\hline
\end{tabular}




\begin{tabular}{|c|c|c|c|c|c|c|c|c|c|c|c|c|c|}
\hline 12 & $\begin{array}{l}0 \\
n \\
n\end{array}$ & $\stackrel{\infty}{n}$ & $\stackrel{n}{I}$ & 党胥 & $\begin{array}{l}\stackrel{\partial}{O} \\
\text { i }\end{array}$ & 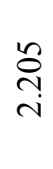 & $\underset{\sim}{\stackrel{J}{N}}$ & $\begin{array}{l}\text { ô } \\
\text { ָె } \\
\text { in }\end{array}$ & $\begin{array}{l}\widetilde{\sigma} \\
\stackrel{0}{+} \\
\dot{0}\end{array}$ & 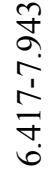 & 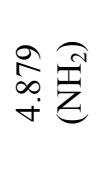 & $\begin{array}{l}n \\
\hat{\sigma} \\
\sigma\end{array}$ & ले \\
\hline 13 & $\stackrel{n}{n}$ & nิ & 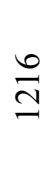 & 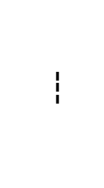 & $\begin{array}{l}\stackrel{+}{\Delta} \\
\text { ते }\end{array}$ & $\stackrel{i}{i}$ & $\begin{array}{l}\stackrel{+}{0} \\
\stackrel{\text { Tे }}{i}\end{array}$ & $\begin{array}{l}\bar{\sigma} \\
\dot{i}\end{array}$ & $\begin{array}{l}\tilde{\sigma} \\
\stackrel{\sim}{*}\end{array}$ & 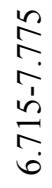 & $\mid$ & $\begin{array}{l}\infty \\
\infty \\
\text { ț }\end{array}$ & $\stackrel{\vec{n}}{i}$ \\
\hline 14 & 离 & రి & $\stackrel{\sim}{\beth}$ & $!$ & $\begin{array}{l}\stackrel{0}{n} \\
i\end{array}$ & $\begin{array}{l}\sqrt[n]{n} \\
\text { in }\end{array}$ & 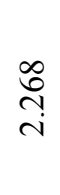 & \begin{tabular}{l}
\multirow{2}{*}{} \\
ô \\
in
\end{tabular} & $\begin{array}{l}\infty \\
0 \\
\stackrel{+}{+}\end{array}$ & 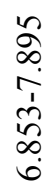 & $\mid$ & $\begin{array}{l}\tilde{1} \\
\tilde{n} \\
0\end{array}$ & $\begin{array}{l}0 \\
\text { in } \\
\text { in }\end{array}$ \\
\hline 15 & $\begin{array}{l}n \\
n \\
n\end{array}$ & $\underset{\mathbb{U}}{\mathbb{J}}$ & $\stackrel{\widetilde{\Xi}}{\beth}$ & $!$ & $\begin{array}{l}n \\
\vdots \\
i\end{array}$ & 오 & $\begin{array}{l}n \\
\text { ñ } \\
i \\
i\end{array}$ & $\begin{array}{l}\sqrt[6]{6} \\
i \\
i\end{array}$ & 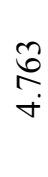 & 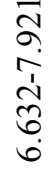 & $\begin{array}{l}\infty \\
\stackrel{n}{n} \\
\dot{n}\end{array}$ & $\begin{array}{l}\text { ले } \\
\text { से }\end{array}$ & $\begin{array}{l}\text { oे } \\
\text { in }\end{array}$ \\
\hline 16 & 疋 & $\begin{array}{l}\infty \\
\stackrel{2}{n}\end{array}$ & $\vec{\Xi}$ & 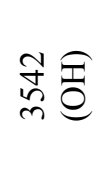 & त् & $\vec{m}$ & $\begin{array}{l}\mathbb{U} \\
\text { तె. } \\
i\end{array}$ & $\frac{\stackrel{+}{n}}{i}$ & $\stackrel{\infty}{\stackrel{\infty}{\sim}}$ & $\begin{array}{l}\vec{\infty} \\
\infty \\
i \\
i \\
\hat{\sim} \\
0 \\
0\end{array}$ & 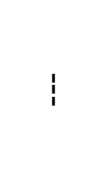 & 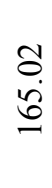 & $\begin{array}{l}\frac{0}{i} \\
\text { in }\end{array}$ \\
\hline 17 & $\begin{array}{l}0 \\
n \\
n \\
m\end{array}$ & $\tilde{\delta}_{0}^{0}$ & $\stackrel{n}{\beth}$ & $\stackrel{\infty}{\stackrel{\Phi}{\beth}}$ & ત્તે & 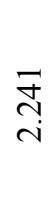 & 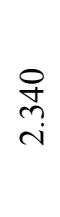 & $\begin{array}{l}\stackrel{0}{0} \\
\stackrel{1}{i}\end{array}$ & $\stackrel{\ddot{m}}{\dot{r}}$ & $\begin{array}{l}\hat{\infty} \\
\stackrel{\infty}{a} \\
\hat{1} \\
\stackrel{0}{\infty} \\
\infty \\
0\end{array}$ & 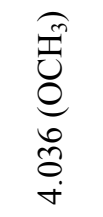 & $\begin{array}{l}\& \\
\text { +ீ}\end{array}$ & $\begin{array}{l}0 \\
\text { in } \\
i n\end{array}$ \\
\hline 18 & $\underset{n}{n}$ & $\stackrel{ }{6}$ & $\stackrel{ }{\mathrm{I}}$ & 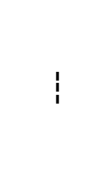 & $\begin{array}{l}\stackrel{0}{\sim} \\
\text { ָे }\end{array}$ & 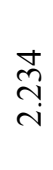 & $\stackrel{\text { }}{\text { ָे }}$ & $\stackrel{n}{\underset{i}{二}}$ & $\begin{array}{l}\stackrel{8}{+} \\
\stackrel{+}{+}\end{array}$ & 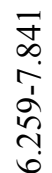 & 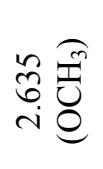 & \begin{tabular}{l}
$\stackrel{\infty}{\sim}$ \\
\multirow{J}{0}{}
\end{tabular} & $\begin{array}{l}\bar{i} \\
i n\end{array}$ \\
\hline 19 & $\begin{array}{l}n \\
n \\
n\end{array}$ & $\frac{n}{6}$ & $\stackrel{n}{\beth}$ & 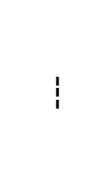 & $\frac{n}{n}$ & 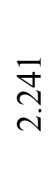 & $\underset{\substack{n \\
i}}{\stackrel{\infty}{n}}$ & $\begin{array}{l}\mathscr{a} \\
\hat{o} \\
i\end{array}$ & 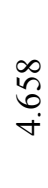 & 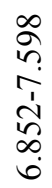 & $\mid$ & 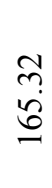 & $\begin{array}{l}\circ \\
\text { ते } \\
\text { nิ }\end{array}$ \\
\hline 20 & $\begin{array}{l}\infty \\
n \\
n \\
n\end{array}$ & 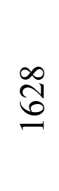 & $\stackrel{\Xi}{\beth}$ & $!$ & $\frac{\circ}{\stackrel{1}{i}}$ & ڤे & $\stackrel{\infty}{\stackrel{+}{\sim}}$ & $\stackrel{+}{\vec{\sigma}}$ & $\begin{array}{l}\stackrel{\infty}{\zeta} \\
\stackrel{+}{+}\end{array}$ & 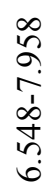 & $\dot{1}$ & 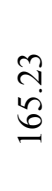 & $\begin{array}{l}\infty \\
\stackrel{\infty}{i} \\
\end{array}$ \\
\hline
\end{tabular}


Table 4(continue). Infrared and NMR spectroscopic data of 4-aryl-5,6-dihydro-6(substituted phenyl)4H-1,3-oxazine-2-amines.

\begin{tabular}{|c|c|c|c|c|}
\hline \multirow[t]{2}{*}{ Entry } & \multicolumn{4}{|c|}{${ }^{13} \mathrm{C}(\delta, \mathrm{ppm})$} \\
\hline & $\mathrm{C}_{5}$ & $\mathrm{C}_{6}$ & $\mathrm{Ar}-\mathrm{C}$ & Substt. \\
\hline 1 & 47.33 & 65.90 & $125.36-142.25$ & --- \\
\hline 2 & 47.98 & 66.25 & $126.25-139.38$ & --- \\
\hline 3 & 47.01 & 65.98 & $122.68-139.25$ & $44.38 \mathrm{~N}\left(\mathrm{CH}_{3}\right)_{2}$ \\
\hline 4 & 48.74 & 65.39 & $121.36-141.25$ & $62.38\left(\mathrm{OCH}_{3}\right)$ \\
\hline 5 & 47.95 & 67.03 & $126.43-139.40$ & --- \\
\hline 6 & 47.94 & 66.79 & $114.54-137.36$ & $56.78\left(\mathrm{OCH}_{3}\right)$ \\
\hline 7 & 47.17 & 66.84 & $125.77-139.04$ & $25.37\left(\mathrm{CH}_{3}\right)$ \\
\hline 8 & 48.26 & 67.25 & $126.37-142.10$ & --- \\
\hline 9 & 47.29 & 66.25 & $124.37-146.02$ & --- \\
\hline 10 & 48.02 & 66.36 & $125.36-146.28$ & --- \\
\hline 11 & 48.62 & 67.25 & $124.29-154.35$ & --- \\
\hline 12 & 47.96 & 66.38 & $121.87-139.25$ & --- \\
\hline 13 & 47.26 & 67.29 & $121.25-138.32$ & --- \\
\hline 14 & 47.29 & 67.28 & $121.35-139.35$ & --- \\
\hline 15 & 47.09 & 67.28 & $121.35-141.29$ & $45.29 \mathrm{~N}\left(\mathrm{CH}_{3}\right)_{2}$ \\
\hline 16 & 47.29 & 66.98 & $118.35-139.32$ & --- \\
\hline 17 & 47.29 & 66.85 & $115.36-158.34$ & $59.57\left(\mathrm{OCH}_{3}\right)$ \\
\hline 18 & 47.21 & 66.28 & $114.28-148.68$ & $24.21\left(\mathrm{CH}_{3}\right)$ \\
\hline 19 & 47.98 & 66.82 & $115.36-159.72$ & --- \\
\hline 20 & 48.09 & 66.28 & $116.38-157.29$ & --- \\
\hline
\end{tabular}

\section{1. Antimicrobial activities}

Antibacterial sensitivity assay of all oxazine amines were performed using Kirby-Bauer [35] disc diffusion technique. In this present investigation the authors have taken B. subtilis, 
M. luteus and $S$. aureus as gram positive E. coli. P.aeruginosa and $K$. pneumoniae as gram negative bacterial strains.

In each Petri plate about $0.5 \mathrm{~mL}$ of the test bacterial sample is spread uniformly over the solidified Mueller Hinton agar using sterile glass spreader. Then the discs with $5 \mathrm{~mm}$ diameter made up of Whatman No.1 filter paper, impregnated with the solution of the compound are placed on the medium using sterile forceps.

The plates are incubated for 24 hours at $37^{\circ} \mathrm{C}$ by keeping the plates upside down to prevent the collection of water droplets over the medium. After 24 hours, the plates are visually examined and the diameter values of the zone of inhibition have been measured. Triplicate results are recorded by repeating the same procedure.

The antibacterial screening effect of synthesized oxazine were shown in (Figure 2; Plates 1-12). The zone of inhibition is compared using Table 5. From the table, it is inferred that the oxazine amines $11,14,17-19$ were shows good activity against B.subtilis. Oxazine amine derivatives 12-14, 17 and 18 were shows good activity against M.luteus. Oxazine amine derivatives 11-15, 18 and 19 were shows good activity against S.aureus. Oxazine amine derivatives $11,13-15$ and 17 were shows good activity against E.coli. Oxazine amine derivatives $11,14,15,17-20$ were shows good activity against $P$.aeruginosa. Oxazine amine derivatives 11-13, 16, 17 and 20 were shows good activity against K.pneumoniae.

Table 5. The antibacterial activities of 4-aryl-5,6-dihydro-6(substituted phenyl)-4H-1,3-oxazine-2amines(entries 11-20).

\begin{tabular}{|c|c|c|c|c|c|c|}
\hline \multirow{3}{*}{ Entry } & \multicolumn{6}{|c|}{ Zone of Inhibition (mm) } \\
\hline & \multicolumn{3}{|c|}{ Gram positive Bacteria } & \multicolumn{3}{|c|}{ Gram negative Bacteria } \\
\hline & B. subtilis & M. luteus & S. aureus & E. coli & P. aeruginosa & K. pneumoniae \\
\hline 11 & 6 & --- & 7 & 6 & 6 & 6 \\
\hline 12 & --- & 6 & 6 & --- & --- & 6 \\
\hline 13 & --- & 6 & 6 & 6 & --- & 6 \\
\hline 14 & 6 & 6 & 6 & 6 & 6 & --- \\
\hline 15 & --- & --- & 6 & 6 & 6 & --- \\
\hline 16 & --- & --- & --- & --- & --- & 6 \\
\hline 17 & 6 & 6 & --- & 6 & 6 & 6 \\
\hline 18 & 6 & 6 & 6 & --- & 6 & --- \\
\hline 19 & 6 & --- & 6 & --- & 6 & --- \\
\hline 20 & --- & --- & --- & --- & 6 & 6 \\
\hline $\begin{array}{c}\text { Standard } \\
\text { Ampicillin }\end{array}$ & 6 & 6 & 7 & 6 & 7 & 7 \\
\hline $\begin{array}{l}\text { Control } \\
\text { DMSO }\end{array}$ & --- & --- & --- & --- & --- & --- \\
\hline
\end{tabular}




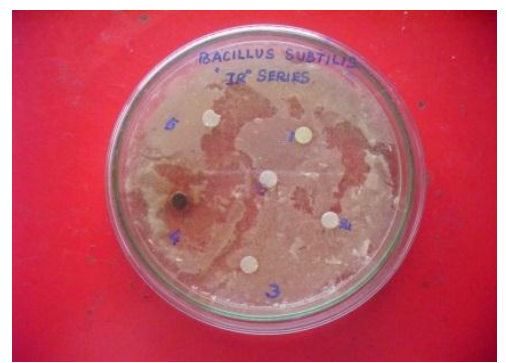

Plate 1

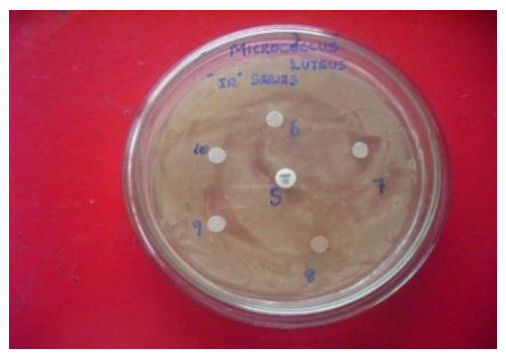

Plate 4

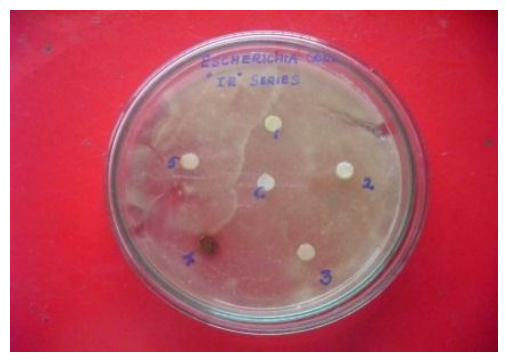

Plate 7

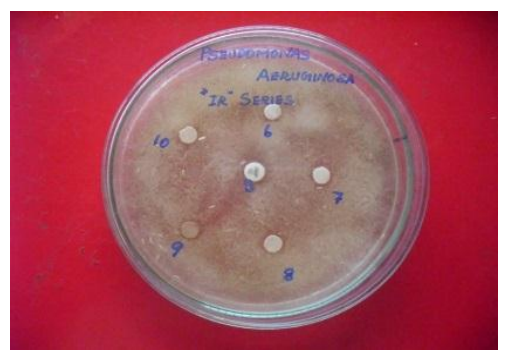

Plate 10

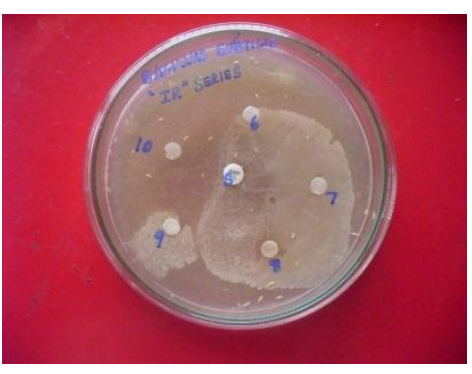

Plate 2

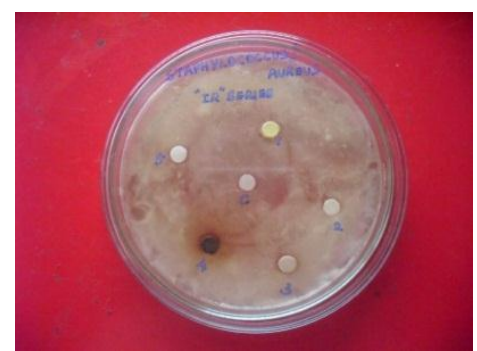

Plate 5

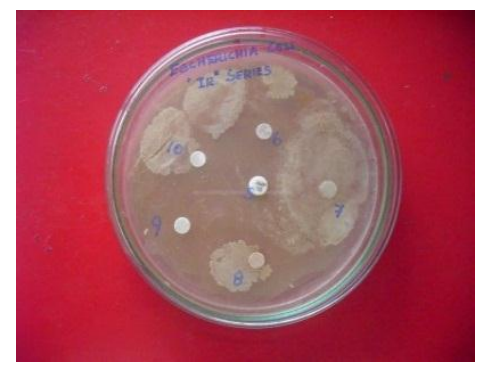

Plate 8

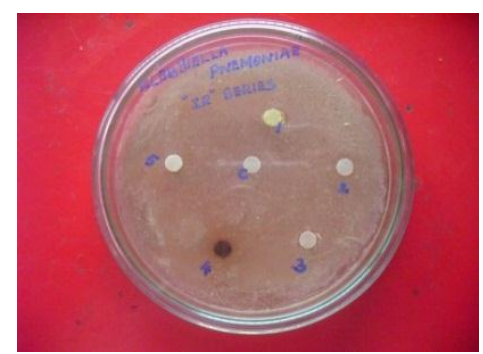

Plate 11

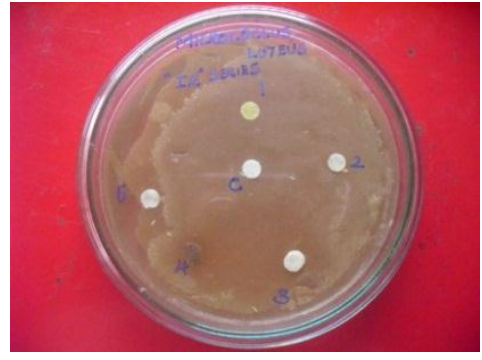

Plate 3

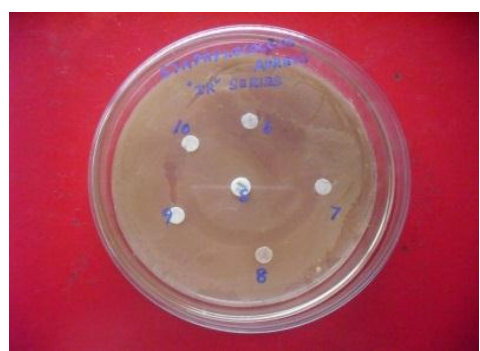

Plate 6

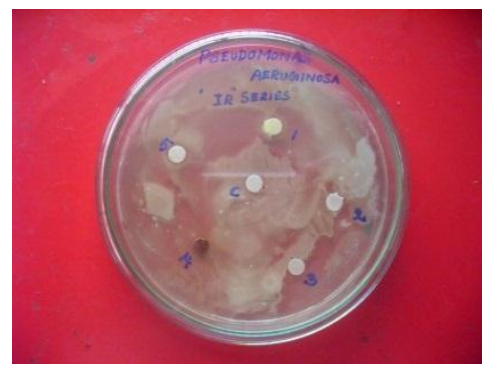

Plate 9

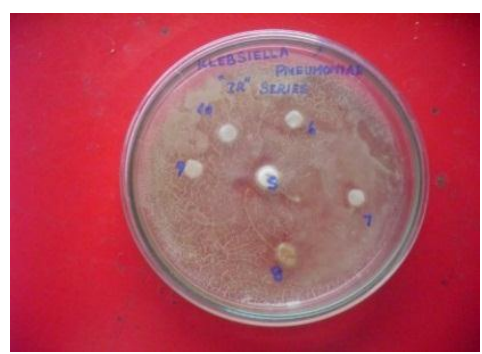

Plate 12

Figure 2. Antibacterial activities of oxazine amine derivatives (entries 11-20): Petri-dishes.

\section{2. Antifungal sensitivity assay}

Antifungal sensitivity assay is performed using Kirby-Bauer [35] disc diffusion technique. The $A$. niger M.species and T. viride fungal strains were employed for evaluating the antifungal activities of synthesised oxazine derivatives. PDA medium is prepared and sterilized as above. It is poured (ear bearing heating condition) in the Petri-plate which is already filled with $1 \mathrm{ml}$ of the fungal species. The plate is rotated clockwise and counter 
clock-wise for uniform spreading of the species. The discs are impregnated with the test solution. The test solution is prepared by dissolving $15 \mathrm{mg}$ of the chalcone in $1 \mathrm{ml}$ of DMSO solvent. The medium is allowed to solidify and kept for 24 hours. Then the plates are visually examined and the diameter values of zone of inhibition have been measured. Triplicate results are recorded by repeating the same procedure.

The antifungal activity of substituted chalcones synthesized in the present study is shown in Figure 3; Plates 1-6 and the zone of inhibition values of the effect is given in Table 6 . From the table the oxazine derivatives $2-4,6$ and 8 shows satisfactory fungal activities against $A$. niger. Compounds 8, 9, 10 and 5-7 were shows excellent good and satisfactory antifungal activities against $E$. coli. The oxazine amines 7, 10 and 2, 3, 5, 8 were shows good and satisfactory antifungal activities against $T$. viride fungal strains.

Table 6. The antifungal activities of 4-aryl-5,6-dihydro-6(substituted phenyl)-4H-1,3-oxazine-2amines (entries 11-20).

\begin{tabular}{|c|c|c|c|}
\hline \multirow{2}{*}{ Entry } & \multicolumn{3}{|c|}{ Zone of Inhibition (mm) } \\
\cline { 2 - 4 } & A. niger & M. species & T. viride \\
\hline 1 & --- & --- & --- \\
\hline 2 & 6 & --- & 6 \\
\hline 3 & 6 & --- & 6 \\
\hline 4 & 6 & --- & --- \\
\hline 5 & --- & 6 & 6 \\
\hline 6 & 8 & 6 & --- \\
\hline 7 & --- & 6 & 7 \\
\hline 8 & 6 & 9 & 6 \\
\hline 9 & --- & 7 & --- \\
\hline 10 & --- & 8 & 10 \\
\hline $\begin{array}{c}\text { Standard } \\
\text { Miconazole }\end{array}$ & 12 & 10 & --- \\
\hline $\begin{array}{c}\text { Control } \\
\text { DMSO }\end{array}$ & --- & --- & \\
\hline
\end{tabular}

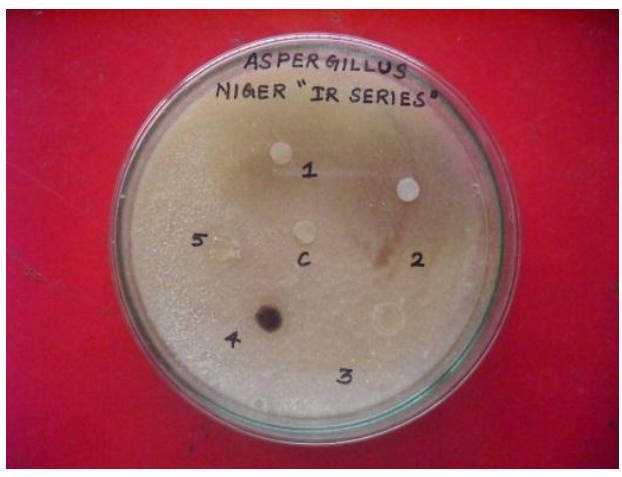

Plate 1

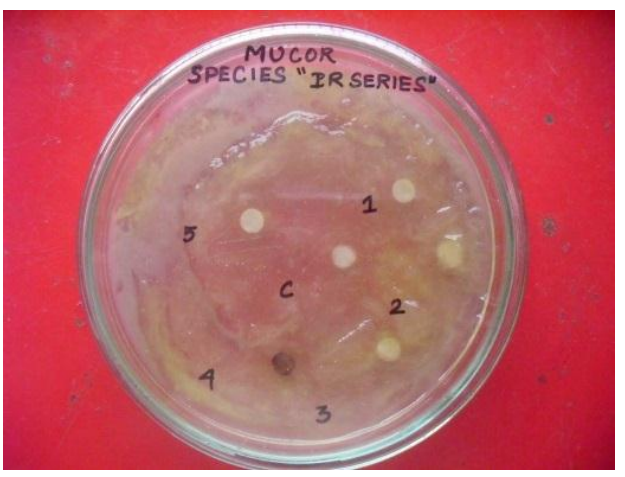

Plate 2 


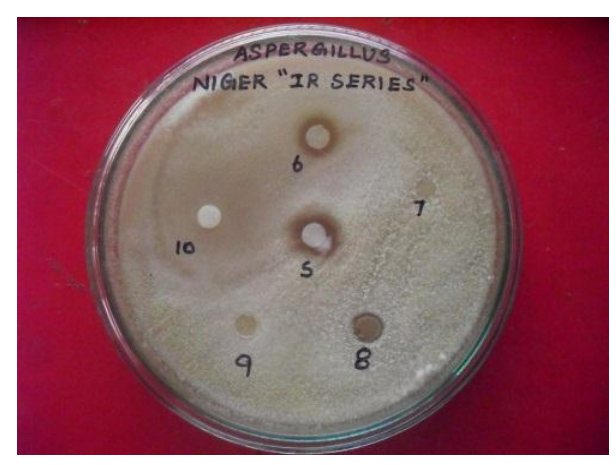

Plate 3

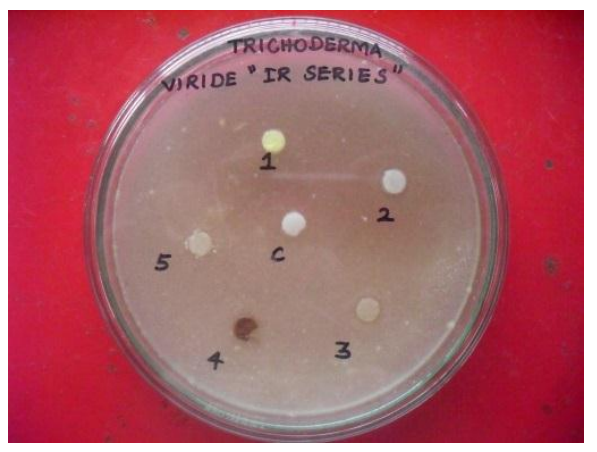

Plate 5

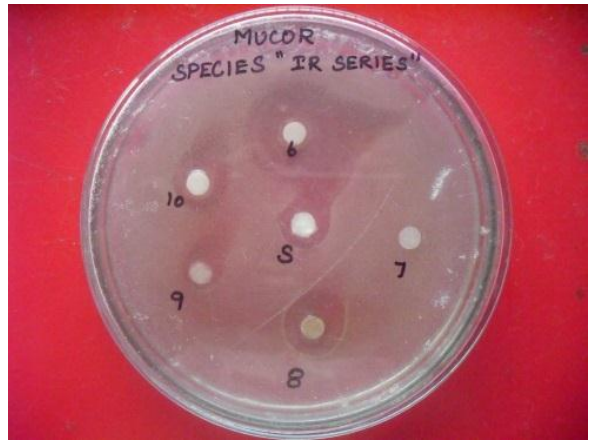

Plate 4

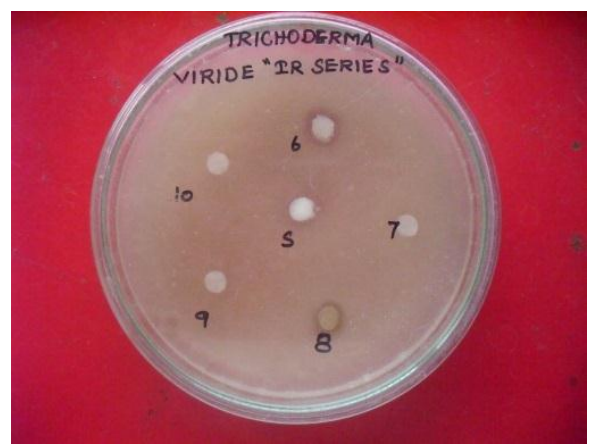

Plate 6

Figure 3. Antifungal activities of oxazine amine derivatives (entries 11-20): Petri-dishes.

\section{CONCLUSIONS}

Some oxazine amine derivatives including $9 H$-flurenyl based oxazine amines have been synthesised by solvent free cyclization of aryl chalcones and urea in presence of flyash: $\mathrm{H}_{2} \mathrm{SO}_{4}$ catalyst under microwave irradiation. This synthetic methodology offers solventfree cyclization, non-hazardous, shorter reaction time, easy-workup procedure and better yields. The analytical and spectral data were supported for these oxazine derivatives. Most of the oxazine derivatives shows good and moderate antimicrobial activities against the respective bacterial and fungal strains.

\section{ACKNOWLEDGEMENT}

The author thank to DST NMR facility, Department of Chemistry, Annamalai university, Annamalainagar608002 for recording NMR spectral of compounds.

\section{References}

[1] I. P. Yakovlev, A. V. Prep'yalov, B. A. Ivin, Chem. Heterocycl. Compd. 30(3) (1994) 255-271.

[2] M. K. Manjula, K. M. L. Rai, S. L. Gaonkar, K. A. Raveesha, S. Satish, Eur. J. Med. Chem. 44 (2009) 280-288. 
[3] B. P. Mathew, A. Kumar, S. Sharma, P. K. Shukla, M. Nath, Eur. J. med. Chem. 45 (2010) 1502-1507.

[4] M. J. Elarfi, H. A. Al-Difar, Sci. Rev. Chem. Communn. 2(2) (2012) 103-107.

[5] V. Tiwari, J. Meshram, P. Ali, J. Sheikh, U. Tripathi, J. Enzyme Inhib. Med. Chem. 26(4) (2011) 569-78.

[6] B. C. Das, A. V. Madhukumar, J. Anguiano, S. Mani, Bioorg. Med. Chem. Lett. 19(15) (2009) 4204-4206.

[7] D. Zhou, B. L. Harrison, U. Shah, T. H. Andree, G. A. Hornby, R. Scerni, Bioorg. Med. Chem. Lett. 16(5) (2006) 1338-1341.

[8] S, Wang, Y. Li, Y. Liu, A. Lu, Q.You, Bioorg. Med. Chem. Lett. 18(14) (2008) 40954097.

[9] Y. Ando, K. Ando, M. Yamaguchi, J. Kunitomo, M. Koida, R. Fukuyama, Bioorg. Med. Chem. Lett. 16(22) (2006) 5849-5854.

[10] L. Bouaziz, P. Nebois, M. H. Bartoli, M. Boitard, H. Fillion, Chem. Pharm. Bull. 44(3) (1996) 605-608.

[11] K. Roy, I. Mitra, A. Saha, Chem. Biol. Drug. Des. 74(5) (2009) 507-516.

[12] A. Blaser, B. D. Palmer, H. S. Sutherland, I. Kmentova, S. G. Franzblau, B. Wan, J. Med. Chem. 55(1) (2012) 312-326.

[13] L. Seal, D. Von Hoff, R. Lawrence, E. Izbicka, R. M. Invest. New Drugs. 15(4), (1997) 289-293.

[14] B. Brudeli, L. R. Moltzau, K. W. Andressen, K. A. Krobert, J. Klaveness, F. O. Levy, Bioorg. Med. Chem. 18(24) (2010) 8600-8613.

[15] M. Akhter, A. Husain, N. Akhter, M. S. Y. Khan, Indian J. Pharm. Sci. 73 (2011) 101104.

[16] D. Gothi, J. M. Joshi, Recent Pat Antiinfect Drug Discov. 6(1) (2011) 27-37.

[17] K. S. Oh, S. Lee, J. K. Choi, B. H. Lee, Comb. Chem. High. Throughput Screen. 13(9) (2010) 790-797.

[18] S. Y. Cho, J. Y. Baek, S. S. Han, S. K. Kang, J. D. Ha, J. H. Ahn, Bioorg. Med. Chem. Lett. 16(3) (2006) 499-502.

[19] S. F. Lee, Q. Vérolet, A. Fürstenberg, Angew. Chem. Inter. Ed. 52(34) (2013) 89488951.

[20] L. A. Kass, Biotech Histochem. 70(1) (1995) 19-23.

[21] L. A. Kass, Biotech Histochem. 70(1) (1995) 29-33.

[22] C. Jung, B. K. Müller, D. C. Lamb, F. Nolde, K. Müllen, C. Bräuchle, J. Am. Chem. Soc. 128(15) (2006) 5283-5291.

[23] J. H. MacMillan, S. S. Washburne, Detailed Synthetic Procedure for 4-(4bromophenyl)-1,3(3H) Oxazine-2,6-Dione and related 4 and 5-aryl substituted -1,3(3H) Oxazine-2,6-Diones. Spectroscopic and analytical data are included. Temple University, http://www.archive.org. 2013.

[24] A. K. Verma, D. Chioudhary, R. K. Saunthwal, V. Rustagi, M. Patel, R. S. Tiwari, J. Org. Chem. 78(13) (2013) 6657-6669. 
[25] G. Thirunarayanan, K. G. Sekar, International Letters of Chemistry, Physics and Astronomy 10(1) (2013) 18-34.

[26] S. Vijayakumar, R. Arulkumaran, R. Sundararajan, S. P. Sakthinathan, R. Suresh, D. Kamalakkannan, K. Ranganathan, K. Sathiyamoorthy, V. Mala, G. Vanangamudi, G. Thirunarayanan, International Letters of Chemistry, Physics and Astronomy 9(1) (2013) 68-86.

[27] M. A. Khalilzadeh, I. Yavari, Z. Hossaini, H. Sadeghifar, Monatsch Chem. 140 (2009) 467-471.

[28] R. Sundararajan, R. Arulkumaran, S. Vijayakumar, D. Kamalakkannan, R. Suresh, S. John Joseph, K. Ranganathan, S. P. Sakthinathan, G. Vanangamudi, G. Thirunarayanan, International Letters of Chemistry, Physics and Astronomy 1 (2014) 67-73.

[29] K. G. Sekar, G. Thirunarayanan, International Letters of Chemistry, Physics and Astronomy 8(3) (2013) 249-258

[30] S. B. Sapkal, K. F. Shelke, A. H. Kategaonkar, M. S. Shingare, Green Chem. Lett. 2(2) (2009) 57-60.

[31] G. Thirunarayanan, K. Ravi, International Letters of Chemistry, Physics and Astronomy 14 (2013) 44-57.

[32] S. P. Sakthinathan, R. Suresh, V. Mala, K.Sathiyamoorthi, D. Kamalakkannan, K. Ranganathan, R. Arulkumaran, S. Vijayakumar, R. Sundararajan, G. Vanangamudi, G. Thirunarayanan, International Letters of Chemistry, Physics and Astronomy 6 (2013) $77-90$.

[33] Z. Turgut, E. Pelit, A.Koycil, Molecules. 12 (2007) 345-352.

[34] G. Thirunarayanan, P. Mayavel, K. Thirumurthy, Spectrochim. Acta 91A (2012) 18-22.

[35] A. W. Bauer, W. M. M. Kirby, J. C. Sherris, M. Truck, Am. J. Clin. Pathol. 45 (1966) 493-496. 